\title{
Nested Saturation Function Control of a Magnetic Levitation System
}

\author{
Octavio Gutiérrez-Frías (iD, Norma Lozada-Castillo (D), J. Alejandro Aguirre-Anaya (iD, \\ and Diego A. Flores-Hernández \\ Instituto Politécnico Nacional-UPIITA, Av. IPN 2580 Col. Barrio La Laguna Ticomán, Ciudad de México, Mexico CP. 07320 \\ Correspondence should be addressed to Octavio Gutiérrez-Frías; ogutierrezf@ipn.mx
}

Received 28 June 2019; Revised 22 January 2020; Accepted 5 February 2020; Published 29 April 2020

Guest Editor: Raúl Villafuerte Segura

Copyright (c) 2020 Octavio Gutiérrez-Frías et al. This is an open access article distributed under the Creative Commons Attribution License, which permits unrestricted use, distribution, and reproduction in any medium, provided the original work is properly cited.

\begin{abstract}
The trajectory tracking task of a magnetic levitation system connected to a beam mechanism is solved by means of a nested saturation control with a feedforward term. The flatness property of the system allows to use the nested saturation control technique and the feedforward control to stabilize the output tracking error around the equilibrium. The closed-loop error dynamics is proven to be locally exponentially stable. Numerical simulations prove the effectiveness of the proposal.
\end{abstract}

\section{Introduction}

Underactuated systems are currently an active research area in automatic control design. On the one hand, their amount of advantages such as lighter structures, economic designs, the possibility of compensating a failure on a fully actuated system, among others, has allowed their use in many applications such as mobile robotics, marine systems, aerospace robotic systems, cranes, flexible robots, and fault compensation systems [1-4]. On the other hand, the control problems of regulation and trajectory tracking of underactuated systems lead to complex solutions which cannot be solved by classical schemes [5]; some of the complexities are due to the difficulties to find general properties concerning their capacity of being linearizable, relations between degrees of freedom and independent control actuators [6], passivity [7, 8], ill-defined relative degrees [9], and so on. Most of the solutions for this class of systems deal with the regulation problem, where energy-based schemes are the most popular. The trajectory planning tasks and their tracking demand more complex strategies. This is due to the fact that not all joint trajectories are attainable for this class of systems [10]. To overcome this problem, the capacity of finding a set of variables that can parameterize the system trajectories (differentially flatness [11-13]) allows to establish a trajectory planning and a subsequent controller synthesis.

In particular, magnetic levitation systems have been extensively analysed and used in the design of frictionless bearings [14], vibration isolation [15], manipulation and micromanipulation systems [16, 17], drug and people transporting $[18,19]$, energy harvesting [20-22], etc., where noncontact motion control or avoiding the use of lubrication systems is desirable [23-25]. The control of this class of systems has been tackled from a wide variety of nonlinear approaches [26, 27]. The magnetic levitation in a beam balance configuration has been approached by feedback linearization [28]. In [29], the regulation problem of a beam balance system is solved though a Lyapunov-based control considering restrictions in states and input. $\mathrm{Hu}$ et al. [30] developed a saturated linear feedback to solve the same problem achieving a larger attraction region with respect to linear techniques. Passivity-based control is also implemented though the interconnection and damping assignment [31]. In [32], a sliding mode controller is used for the regulation problem using two magnetic actuators in an agonist-antagonist configuration. The problem of trajectory tracking for a ball levitation system controlled by magnetic 
bearings has been solved by a flatness-based control [33-35], where path planning is used to avoid the input constraints in the control design although the natural saturations are not part of the control design in contrast with other schemes. The idea of using the saturations as a part of the control design in combination with the advantages of a flatnessbased control seems to be a good alternative to solve the problem of trajectory tracking enhancing the control response in case the control saturation may arise. The nested saturation function approach was introduced by A. Teel in [36] to solve the stabilization of a chain of integrator of arbitrary order, and it can also be used for trajectory tracking for a class of trajectories restricted. Other works that solve this problem are presented in [37-39]. Besides, this technique has been used for stabilization of a class of underactuated systems [40-43].

In this sense, this article deals with the problem of trajectory tracking of a beam balance levitation system by means of a nested saturation function approach in combination with the flatness property of the system. The proposal consists in using the flatness of the system to transform the system in an integrator chain, and then, a controller based on a nested saturated function and a feedforward term is used to force the output trajectories to converge towards the reference trajectory. The closed-loop solution is proven to be locally exponentially stable by means of the second method of Lyapunov.

The remainder of the article is given as follows: the dynamical model of the magnetic levitation system, the flatness property, and the problem formulation are given in Section 2. The control proposal and its stability proof are provided in Section 3. Some numerical simulations which show the behaviour of the proposal are presented in Section 4 , and finally, some concluding remarks are stated.

\section{System Model}

Consider the magnetic levitation system, shown in Figure 1, where a beam can rotate freely, and its movement is affected by the gravity effects and the control input derived from a magnetic force generated on a controlled electromagnet coil. The dynamical model is given as follows:

$$
\begin{aligned}
& \dot{x}_{1}(t)=x_{2}(t), \\
& \dot{x}_{2}(t)=\frac{C e}{J}\left[\frac{x_{3}(t)}{l \sin \left(x_{1}(t)\right)}\right]^{2}-\frac{m g r \cos \left(x_{1}(t)\right)}{J}, \\
& \dot{x}_{3}(t)=\frac{u(t)}{L}-\frac{R x_{3}(t)}{L},
\end{aligned}
$$

where $x_{1}(t)$ is the angular position of the pendulum, $x_{2}(t)$ is its corresponding angular velocity, and $x_{3}(t)$ is the current of the inductor. Ce represents the electromagnetic force proportionality constant. $J$ is the inertia moment of the beam, $l$ is the distance between the pivot and the center of the permanent magnet, $m$ denotes the mass of the beam, $g$ is the gravity constant, and $r$ is the radial distance of the center of mass of the beam to the rotational center. $L$ and $R$ are the

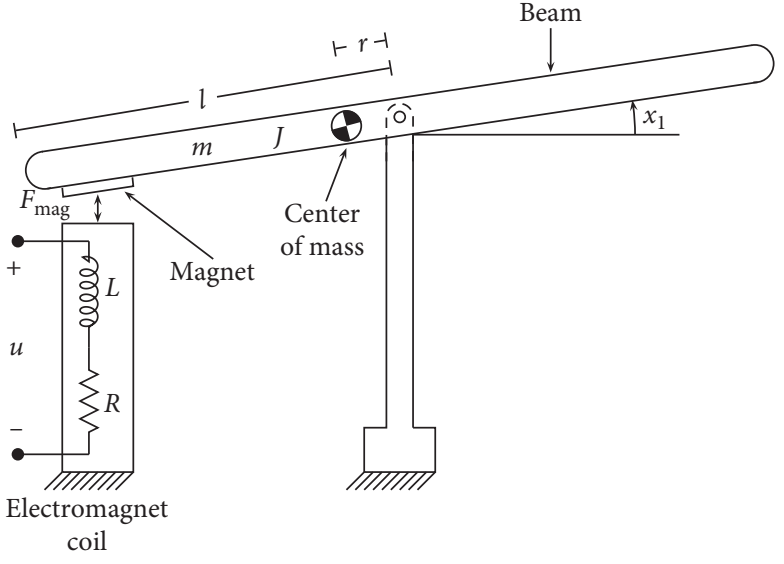

Figure 1: Magnetic levitation system.

inductance and resistance parameters of the electromagnetic subsystem. Finally, $u(t)$ is the control input, applied on the electromagnetic system. It is assumed that the output variable is the position of the beam $x_{1}(t)$.

Last system admits a canonical controllable form by means of the following change of coordinates [44]:

$$
\begin{aligned}
& F_{1}(t)=x_{1}(t), \\
& F_{2}(t)=x_{2}(t), \\
& F_{3}(t)=\alpha_{1}\left(\frac{x_{3}(t)}{\sin \left(x_{1}(t)\right)}\right)^{2}-\alpha_{2} \cos \left(x_{1}(t)\right),
\end{aligned}
$$

where $\alpha_{1}=\left(\mathrm{Ce} / J l^{2}\right)$ and $\alpha_{2}=(m g r / J)$.

We have the following inverse transform:

$$
\begin{aligned}
& x_{1}(t)=F_{1}(t) \\
& x_{2}(t)=F_{2}(t) \\
& x_{3}(t)=\sqrt{\frac{1}{\alpha_{1}}\left(F_{3}(t)+\alpha_{2} \cos \left(F_{1}(t)\right)\right) \sin ^{2}\left(F_{1}(t)\right)} .
\end{aligned}
$$

The dynamics of (1) in terms of coordinate transformation (2) is given as follows:

$$
\begin{aligned}
\dot{F}_{1}(t)= & F_{2}(t), \\
\dot{F}_{2}(t)= & F_{3}(t), \\
\dot{F}_{3}(t)= & -2\left(F_{3}(t)+\alpha_{2} \cos \left(F_{1}(t)\right)\right) r\left(F_{1}(t), F_{2}(t)\right) \\
& +\alpha_{2} \sin \left(F_{1}(t)\right) F_{2}(t) \\
& +\frac{2 \sqrt{\alpha_{1}}}{L} \sqrt{\frac{F_{3}(t)+\alpha_{1} \cos \left(F_{1}(t)\right)}{\sin ^{2}\left(F_{1}(t)\right)}} u(t)
\end{aligned}
$$

where

$$
r\left(F_{1}(t), F_{2}(t)\right)=\left(\frac{R}{L}+F_{2}(t) \cot \left(F_{1}(t)\right)\right) .
$$


This system can be controllable except when $F_{1}(t)=0$ or in the set $F_{3}(t)+\alpha_{1} \cos \left(F_{1}(t)\right)=0$.

2.1. Problem Formulation. The following problem formulation is established: given a smooth admissible reference trajectory of the beam angle, denoted as $x_{1}^{*}(t)$, devise an output feedback control law $u(t)$ such that the trajectory tracking error remains after a settling time on a vicinity of the origin of the phase variable plane, as small as allowed by the system nonlinearities and the control gains to be designed.

\section{Nested Function Control Design}

From (4), it can be shown that the system is differentially flat $[11,12]$ with flat output $F_{1}(t)=x_{1}(t)$. That is, system (4) has the form

$$
F_{(1)}^{(3)}(t)=h\left(F_{1}(t), \dot{F}_{1}(t), \ddot{F}_{1}(t)\right)+p\left(F_{1}(t), \ddot{F}_{1}(t)\right) u(t),
$$

with

$$
\begin{array}{r}
h\left(F_{1}(t), \dot{F}_{1}(t), \ddot{F}_{1}(t)\right)=-2\left(\ddot{F}_{1}(t)+\alpha_{2} \cos \left(F_{1}(t)\right)\right) \times \\
\left(\frac{R}{L}+\dot{F}_{1}(t) \cot \left(F_{1}(t)\right)\right)+\alpha_{2} \sin \left(F_{1}(t)\right) \dot{F}_{1}(t) \\
p\left(F_{1}(t), \ddot{F}_{1}(t)\right)=\frac{2 \sqrt{\alpha_{1}}}{L} \sqrt{\frac{\ddot{F}_{1}(t)+\alpha_{1} \cos \left(F_{1}(t)\right)}{\sin ^{2}\left(F_{1}(t)\right)} .}
\end{array}
$$

The following linearizing control input

$$
u(t)=\frac{1}{p\left(F_{1}(t), \ddot{F}_{1}(t)\right)}\left(-h\left(F_{1}(t), \dot{F}_{1}(t), \ddot{F}_{1}(t)\right)+\bar{v}(t)\right),
$$

yields the following linear controllable in the Brunovsky canonical form

$$
F_{(1)}^{(3)}(t)=\bar{v}(t)
$$

Let us define the flat output error as $e_{F 1}(t):=F_{1}(t)-F_{1}^{*}(t)$, where $F_{1}^{*}(t)=x_{1}^{*}(t)$. In order to express the dynamics in terms of the tracking error coordinates, the following feedforward input term is introduced in the last expression:

$$
\bar{v}(t)=F_{1}^{*(3)}(t)+v(t) .
$$

Thus, using (8) and (10) in (4), the following expression is obtained:

$$
e_{F 1}^{(3)}(t)=v(t)
$$

Last system admits the following canonical representation:

$$
\begin{aligned}
& \dot{e}_{F 1}(t)=e_{F 2}(t), \\
& \dot{e}_{F 2}(t)=e_{F 3}(t), \\
& \dot{e}_{F 3}(t)=v(t) .
\end{aligned}
$$

Let us introduce the following linear transformation $[36,45]$ :

$$
\left[\begin{array}{l}
q_{1}(t) \\
q_{2}(t) \\
q_{3}(t)
\end{array}\right]=\left[\begin{array}{lll}
1 & 2 & 1 \\
0 & 1 & 1 \\
0 & 0 & 1
\end{array}\right]\left[\begin{array}{l}
e_{F 1}(t) \\
e_{F 2}(t) \\
e_{F 3}(t)
\end{array}\right] .
$$

Last transformation leads system (12) to

$$
\begin{aligned}
& \dot{q}_{1}(t)=q_{2}(t)+q_{3}(t)+v(t), \\
& \dot{q}_{2}(t)=q_{3}(t)+v(t), \\
& \dot{q}_{3}(t)=v(t) .
\end{aligned}
$$

Therefore, as system (12) is expressed with a chain of integrator, we suggested a controller based on nested saturation function. This technique, proposed in [36], has been used for controlling a wide class of the underactuated system [40-42, 46]. Thus, our stability problem will be solved as follows. First, a linear transformation is used to directly propose a trajectory tracking controller; then, it is shown that the proposed controller guarantees the boundedness of all states, and after a finite time, it is possible to ensure that all states converge to zero [43].

Let us define the following saturation function:

Definition 1. The linear saturation function $\sigma_{m}(s): \mathbb{R} \longrightarrow \mathbb{R}$ is defined as

$$
\sigma_{m}(s)=\left\{\begin{array}{lll}
s, & \text { if } & |s| \leq m, \\
m \cdot \operatorname{sign}(s), & \text { if } & |s|>m .
\end{array}\right.
$$

Finally, the trajectory tracking controller can be proposed as

$$
v(t)=-q_{3}(t)-K \sigma_{\alpha}\left(\frac{q_{2}(t)+\sigma_{\beta}\left(q_{1}(t)\right)}{K}\right),
$$

where $K$ is a positive constant, $\alpha>0$ and $\beta>0$ are fixed parameters, and $\sigma_{m}$ is a linear saturation function.

3.1. Boundedness of All States. We show in three simple steps that the closed-loop solution of the proposed closed-loop systems (14) and (16) ensures that all the states are bounded. Moreover, the bound of each state directly depends on the designed parameters of the controller.

Step 1. We define a positive definite function

$$
V_{3}\left(q_{3}, t\right)=\frac{q_{3}^{2}(t)}{2}
$$

Then, differentiating (17) and using the third differential equation of (14), we have the time derivative of $V_{3}\left(q_{3}, t\right)$ given by 


$$
\dot{V}_{3}\left(q_{3}, t\right)=-q_{3}^{2}(t)-q_{3}(t) K \sigma_{\alpha}\left(\frac{q_{2}(t)+\sigma_{\beta}\left(q_{1}(t)\right)}{K}\right) \text {. }
$$

It is clear that $\dot{V}_{3}\left(q_{3}, t\right)<0$ when $\left|q_{3}(t)\right| \geq \alpha K$; therefore, there is a finite time $T_{1}>0$ such that

$$
\left|q_{3}(t)\right|<\alpha K, \quad \forall t>T_{1} \text {, }
$$

where $K>0$.

Step 2. Now, we proceed to analyze the behaviour of the state $q_{2}(t)$. Hence, we introduce a positive definite function $V_{2}\left(q_{2}, t\right)=q_{2}^{2}(t) / 2$. Differentiating $V_{2}\left(q_{2}, t\right)$, we obtain after substituting (16) into second differential equation of (14)

$$
\dot{V}_{2}\left(q_{2}, t\right)=-q_{2}(t) K \sigma_{\alpha}\left(\frac{q_{2}(t)+\sigma_{\beta}\left(q_{1}(t)\right)}{K}\right),
$$

where $\alpha$ and $\beta$ are selected such that $\alpha>2 \beta$. Evidently, if $\left|q_{2}(t)\right|>\beta$, then $\dot{V}_{2}\left(q_{2}, t\right)<0$, and there is finite $T_{2}>T_{1}$ after which

$$
\left|q_{2}(t)\right|<\beta, \quad \forall t>T_{2},
$$

when this condition is satisfied, and the control $v(t)$ turns out to be

$$
v(t)=-q_{3}(t)-q_{2}(t)-\sigma_{\beta}\left(q_{1}(t)\right) .
$$

Step 3. Substituting (22) into first differential equation of (14), we obtain

$$
\dot{q}_{1}(t)=-\sigma_{\beta}\left(q_{1}(t)\right)
$$

Now, we define an auxiliary positive definite function $V_{1}\left(q_{1}, t\right)=q_{1}^{2}(t) / 2$. By differentiating $V_{1}\left(q_{1}, t\right)$ along the trajectories of (23), we obtain

$$
\dot{V}_{1}\left(q_{1}, t\right)=-q_{1}(t) \sigma_{\beta}\left(q_{1}(t)\right)
$$

where $\beta$ must be chosen such that $\beta>0$. If $\left|q_{1}(t)\right|>0$, then $\dot{V}_{1}\left(q_{1}, t\right)<0$, and hence, there is finite $T_{3}>T_{2}$ after which

$$
\left|q_{1}(t)\right|<0, \quad \forall t>T_{3} .
$$

Consequently, $q_{1}$ is also bounded. So, all previous constraints on parameters $\alpha$ and $\beta$ can be summarized as

$$
\begin{gathered}
\alpha>2 \beta, \\
\beta>0 .
\end{gathered}
$$

Manipulating the last inequalities, we can select the control parameters as follows:

$$
\begin{aligned}
& \alpha=2 \lambda, \\
& \beta=\lambda,
\end{aligned}
$$

where $\lambda>0$.

3.2. Convergence of All States to Zero. We shall prove that the closed-loop system given by (14) and (16) is asymptotically stable and locally exponentially stable, provided that the controller parameters satisfy (26).

We must note that, after $t>T_{3}$, the control law is no longer saturated, that is,

$$
v(t)=-q_{3}(t)-q_{2}(t)-q_{1}(t),
$$

and the closed-loop system can be expressed as

$$
\begin{aligned}
& \dot{q}_{1}(t)=-q_{1}(t), \\
& \dot{q}_{2}(t)=-q_{2}(t)-q_{3}(t), \\
& \dot{q}_{3}(t)=-q_{3}(t)-q_{2}(t)-q_{1}(t) .
\end{aligned}
$$

Let us define the following Lyapunov function:

$$
V(q, t)=\frac{1}{2} q(t)^{\top} q(t)
$$

with $q(t):=\left[\begin{array}{lll}q_{1}(t) & q_{2}(t) & q_{3}(t)\end{array}\right]^{\top}$. Now, differentiating $V(q, t)$ along the trajectories of (29), we obtain

$$
\dot{V}(q, t)=-q(t)^{\top} M q(t),
$$

where

$$
M=\left[\begin{array}{ccc}
1 & 1 / 2 & 1 / 2 \\
1 / 2 & 1 & 1 / 2 \\
1 / 2 & 1 / 2 & 1
\end{array}\right] .
$$

Note that $\lambda_{\min }\{M\}=1 / 2$, and therefore, $M$ is positive definite Therefore, $\dot{V}(q, t)$ is negative definite, and the vector state $q$ locally exponentially converges to zero after $t>T_{3}$.

From the above discussion, we have the following.

Proposition 1. Consider the magnetic levitation system as described (1) in a closed loop with controller (8). Then, the closed-loop system is asymptotically stable and locally exponentially stable provided that the control parameters $\alpha$ and $\beta$ satisfy the inequalities.

\section{Numerical Simulations}

To test the performance of the controller, we carried out some numerical simulations using MATLAB program, and the results were obtained based on the numerical method of Runge-Kutta of fourth order with the fixed step of $0.001 \mathrm{~s}$. The physical parameters of the system are $C_{e}=9.9081 \times^{-6}\left(\mathrm{Nm}^{2} / \mathrm{A}^{2}\right), J=0.01\left(\mathrm{~kg} \mathrm{~m}^{2}\right), l=0.2(\mathrm{~m}), m=$ $0.6(\mathrm{~kg}), g=9.81\left(\mathrm{~m} / \mathrm{s}^{2}\right), r=0.009(\mathrm{~m}), L=0.2703(\mathrm{H})$, and $R=4.5(\Omega)$, and the controller parameter values were set as $\alpha=3, \beta=1.5$, and $K=1$.

The first experiment shows the behaviour of the system, for a rest-to-rest trajectory, and in this case, we use a reference trajectory-type Bezier polynomial $\left(x_{1}^{*}(t)\right)$. The initial conditions were set as $x_{1}(0)=-0.1(\mathrm{rad}), x_{2}(0)=0(\mathrm{rad} / \mathrm{s})$, and $x_{3}(0)=0.5(\mathrm{~A})$. In Figure 2, the output tracking response is shown, and we can see that the controller effectively brings the system from initial position $\left(x_{1}(0)=-0.1(\mathrm{rad})\right)$ to the final rest position $\left(x_{1}^{*}\left(t_{f}\right)=0.4\right)$ after 7 seconds. The behaviour for the states $x_{2}(t)$ and $x_{3}(t)$ is shown in Figure 3. Finally, Figure 4 depicts the control input. 


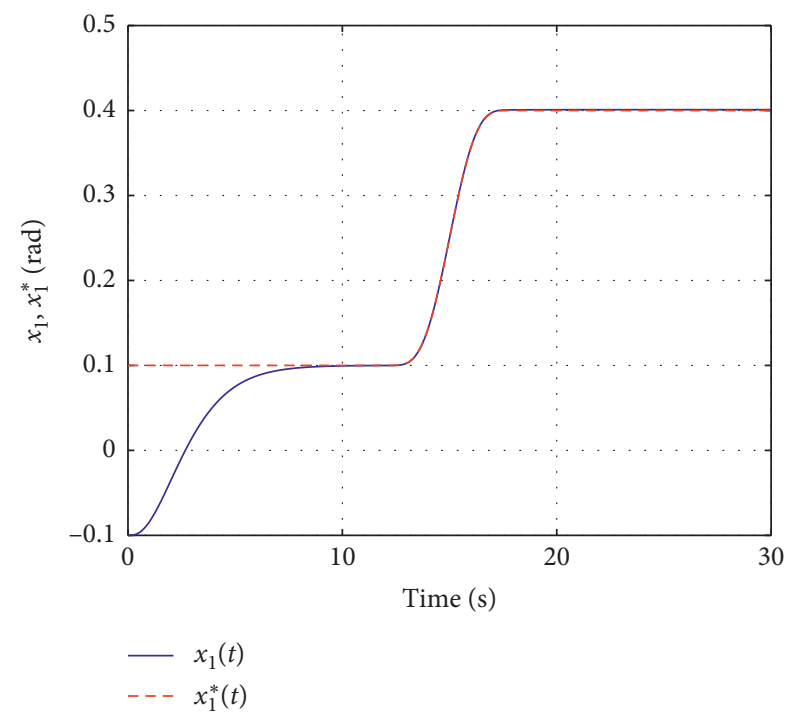

FIGURE 2: Output tracking response for a rest-to-rest trajectory.

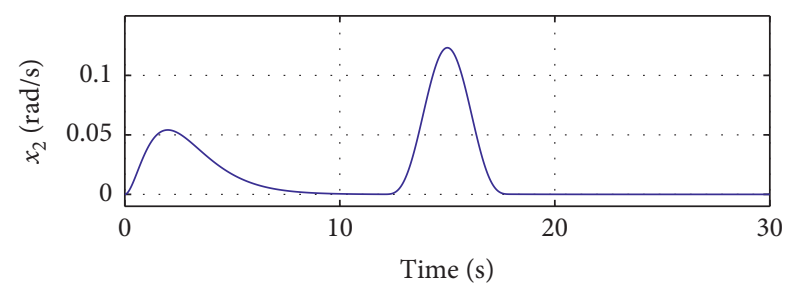

$-x_{2}(t)$

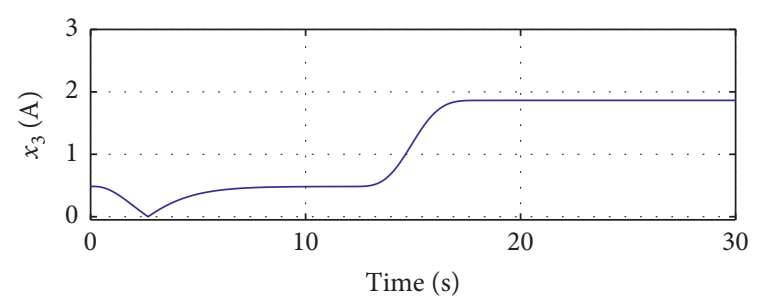

$-x_{3}(t)$

(a)

(b)

FIGURE 3: State behaviour in a rest-to-rest trajectory.

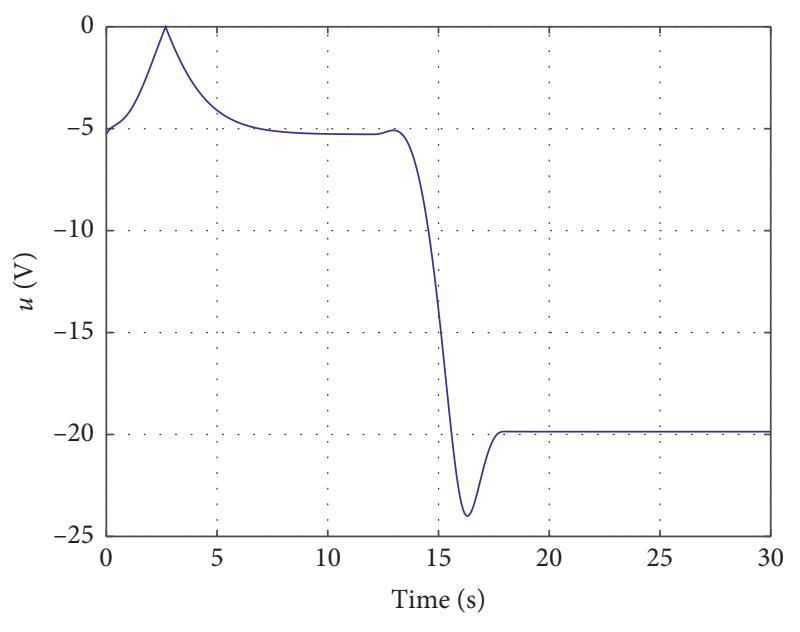

$-u(\mathrm{t})$

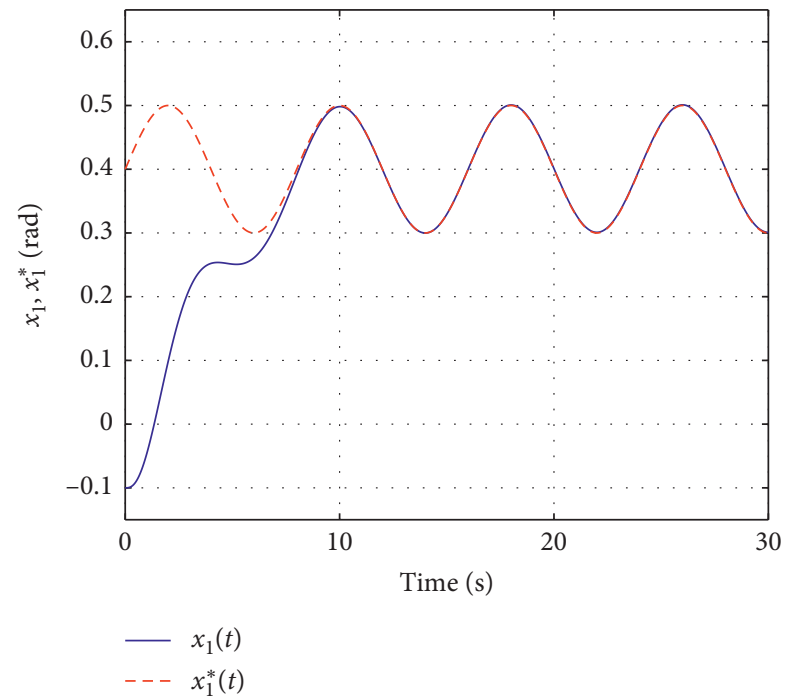

FIGURE 5: Output tracking response for a sinusoidal trajectory. 


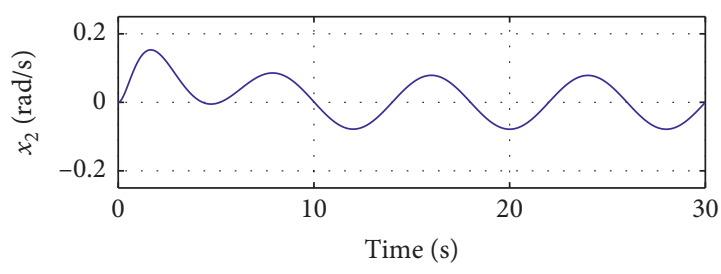

$-x_{2}(t)$

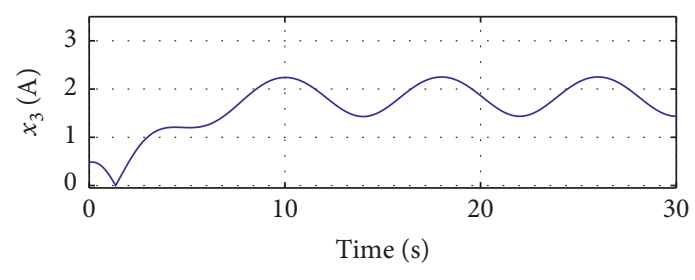

$-x_{3}(t)$

(a)

(b)

FIGURE 6: State behaviour in a sinusoidal trajectory.

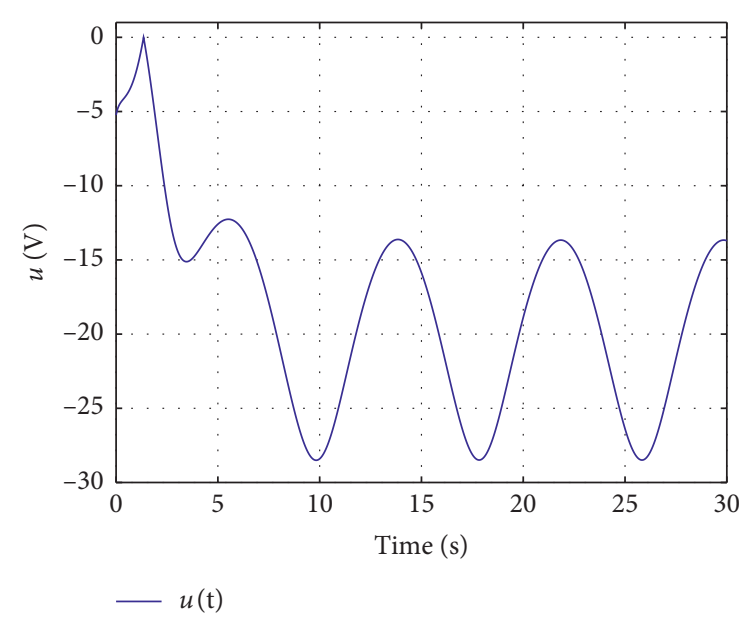

FIgURE 7: Control input (sinusoidal reference).

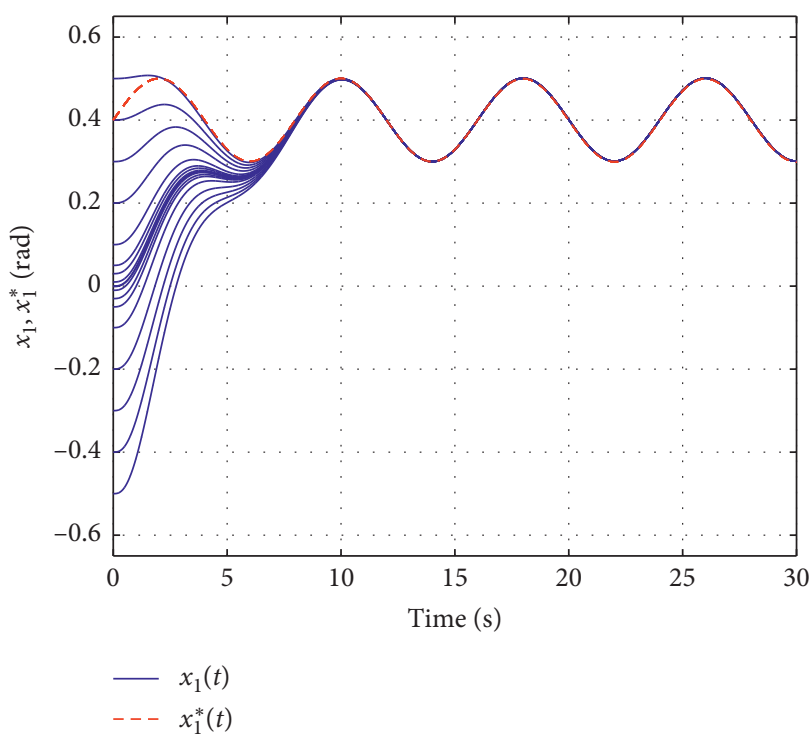

Figure 8: Output tracking response for a sinusoidal trajectory under different initial conditions.

The second experiment was designed under the same setup, but in this case, we use a reference sinusoidal trajectory $\left(x_{1}^{*}(t)=0.4+0.1 \sin (\pi t / 4)\right)$. The result of the

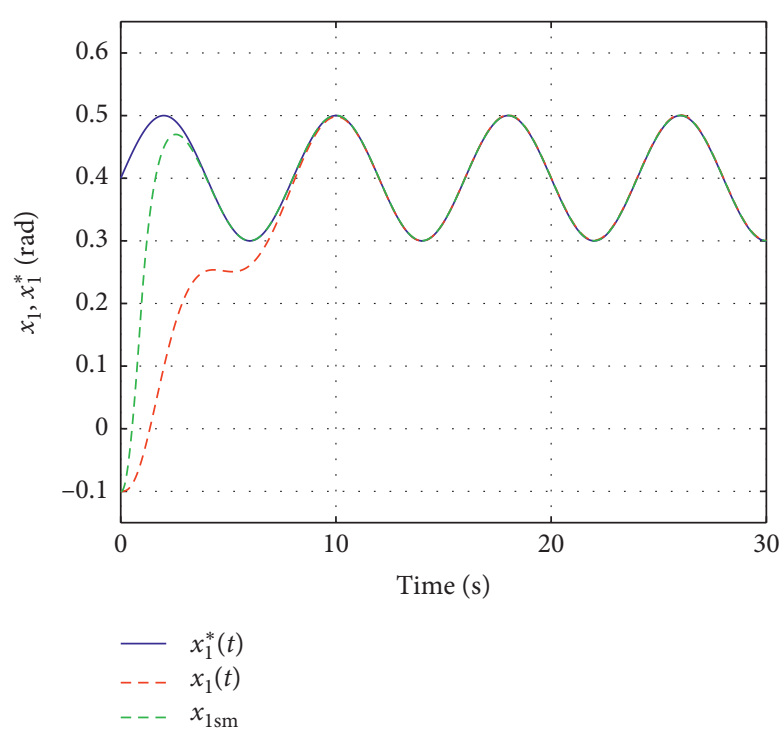

Figure 9: Comparison results for sinusoidal trajectory tracking.

experiment is presented in Figure 5. From this figure, we can see that the controller was capable of performing the task of tracking a sinusoidal trajectory. In Figure 6, the behaviour for the states $x_{2}(t)$ and $x_{3}(t)$ is shown. Finally, Figure 7 presents the control input.

In Figure 8, the output tracking response for a sinusoidal trajectory with different initial conditions is shown. In this case, $x_{1}(0)=[-0.5,0.5](\mathrm{rad})$, and we can see that the controller was capable of performing the task of tracking a sinusoidal trajectory adequately. Some tests were carried out in order to verify the sensitivity of the control scheme with respect to the initial conditions, in which a set of different initial conditions in an admissible operation range was used. The results indicate that the response is not affected by the initial conditions, and there is a lack of overshooting effects which are typical in highgain schemes.

4.1. A Comparison Test. In order to assess the behaviour of the control proposal, a comparison test was made against a robust control strategy, consisting in a sliding mode controller (see [47]). The sliding mode controller was set to be 


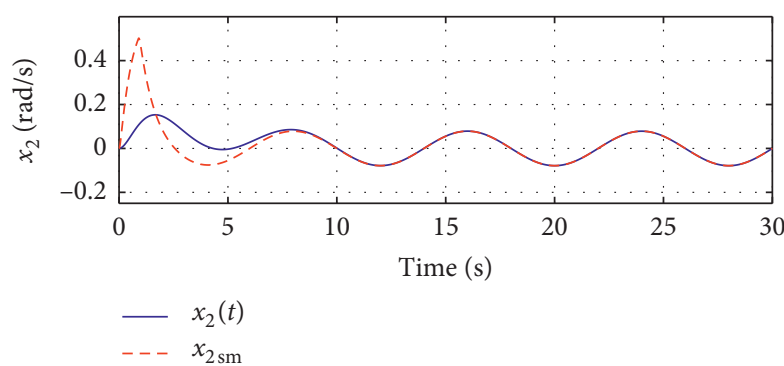

(a)

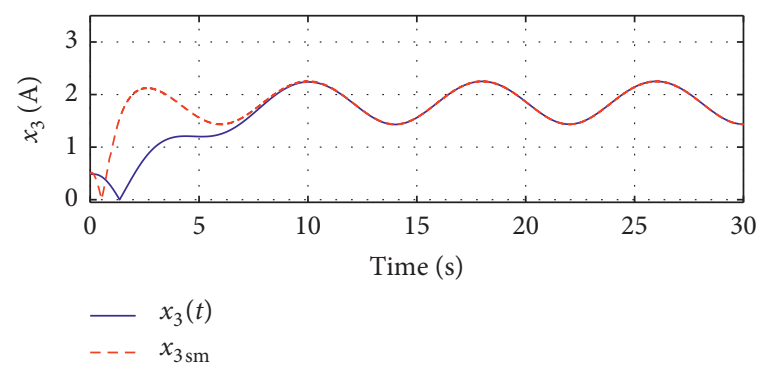

(b)

Figure 10: State behaviour (comparison test).

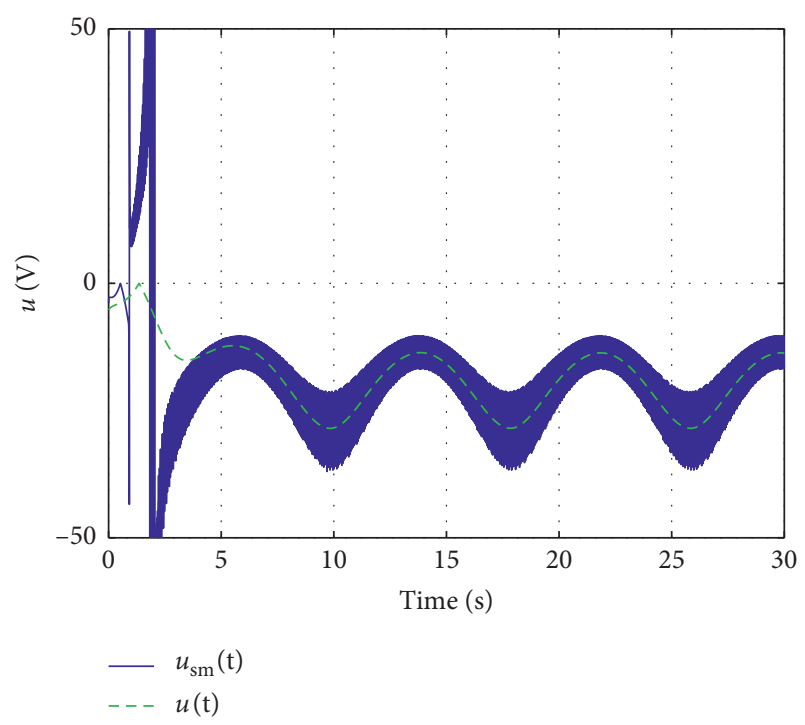

FIgURE 11: Control input (comparison test).

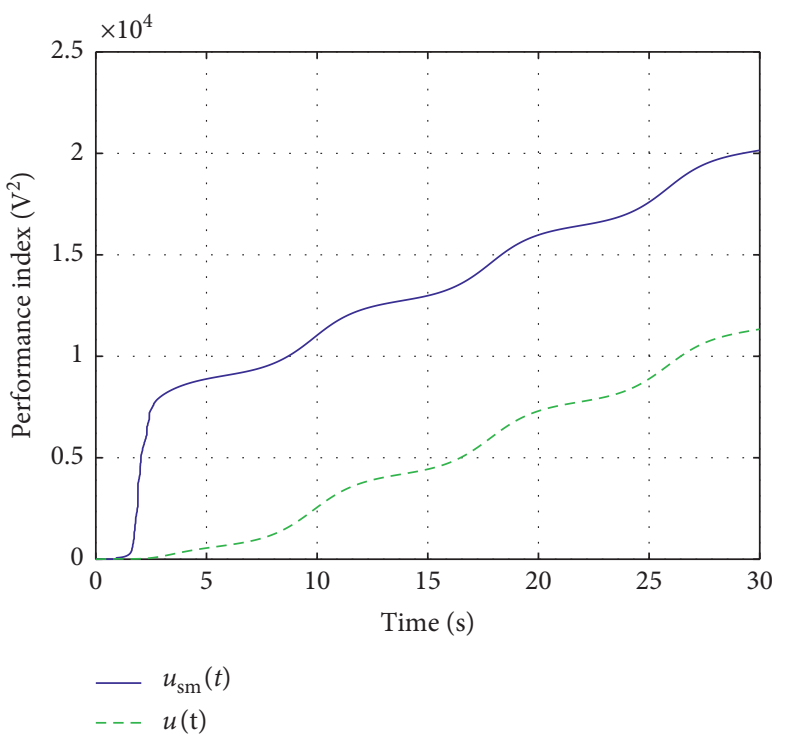

Figure 12: Performance index of the input control.

$$
\begin{aligned}
& u(t)=\frac{1}{p\left(F_{1}(t), \ddot{F}_{1}(t)\right)}\left(-h\left(F_{1}(t), \dot{F}_{1}(t), \ddot{F}_{1}(t)\right)+\bar{v}_{\mathrm{sm}}(t)\right), \\
& \bar{v}_{\mathrm{sm}}(t)=F_{1}^{*(3)}-\lambda_{2} e_{F 3}-\lambda_{1} e_{F 2}-W \operatorname{sign}(\sigma), \\
& \sigma=e_{F 3}+\lambda_{2} e_{F 2}+\lambda_{1} e_{F 1}, \\
& \text { with }: \lambda_{2}, \lambda_{1} \in \mathbb{R}^{+} .
\end{aligned}
$$

The control parameters used in the test were $W=20$, $\lambda_{2}=24$, and $\lambda_{1}=36$. Figure 9 shows the tracking results, where the sliding mode results (denoted by the subindex sm) converged faster without any overshooting effects. Figure 10 depicts the states $x_{2}$ and $x_{3}$, in which both schemes had similar values (reachable for experimental implementations). Figure 11 shows the advantages of the proposal since the voltage input of the sliding mode showed the high-frequency behaviour, which is a classic problem in sliding mode control implementations which may demand high control efforts. Notice that the proposal has a better energy management, leading to similar results with a smoother control input. This can be noticed with the obtained performance index $\int u^{2}$ which is shown in Figure 12.

\section{Conclusion}

A nested saturation-based controller for the trajectory tracking task on a beam levitation system was introduced and proven to be effective. The flatness property of the magnetic levitation system permits to express the system as a third-order integrator chain, allowing the use of nested saturation functions to design a trajectory tracking controller.

The proposed scheme forces the system output to track the reference trajectory even in the presence of the input constraints and ensures that all states converge exponentially to the desired trajectory. The stability analysis is based on the second method of Lyapunov using a simple candidate function. Some computer simulations showed the effectiveness of the proposal in the tracking of a rest-to-rest and sinusoidal trajectories. 
On the contrary, as a future research, some other nonlinearities such as hysteresis in the magnetic actuator [48] can be addressed. This effect is important for the design of maglev systems in superconductor-based transportation system applications.

\section{Data Availability}

No data were used to support this study.

\section{Conflicts of Interest}

The authors declare that there are no conflicts of interest regarding the publication of this article.

\section{Acknowledgments}

This article was supported by the Secretaría de Investigación y Posgrado of the Instituto Politécnico Nacional (SIP-IPN) under the research grants 20195901, 20196059, 20196079, 20196855 ,20201768, 20201829 and 20201830. J. Alejandro Aguirre-Anaya is a scholarship holder of the CONACYT.

\section{References}

[1] J. Moreno-Valenzuela and C. Aguilar-Avelar, Motion Control of Underactuated Mechanical Systems, Springer, Berlin, Germany, 2018.

[2] S. Rudra, R. K. Barai, and M. Maitra, Block Backstepping Design of Nonlinear State Feedback Control Law for Underactuated Mechanical Systems, Springer, Berlin, Germany, 2017.

[3] R. Olfati-Saber, Nonlinear control of underactuated mechanical systems with application to robotics and aerospace vehicles, Massachusetts Institute of Technology, Cambridge, MA, USA, Ph.D. dissertation, 2001.

[4] M. Spong, "Underactuated mechanical systems," in Control Problems in Robotics and Automation, pp. 135-150, Springer, Berlin, Germany, 1998.

[5] A. Choukchou-Braham, B. Cherki, M. Djemai, and K. Busawon, Analysis and Control of Underactuated Mechanical Systems, Springer Science \& Business Media, Berlin, Germany, 2013.

[6] G. Oriolo and Y. Nakamura, "Control of mechanical systems with second-order nonholonomic constraints: underactuated manipulators," in Proceedings of the 30th IEEE Conference on Decision and Control, pp. 2398-2403, IEEE, Brighton, UK, December 1991.

[7] R. Ortega, A. Loría, P. Nicklasson, and H. Sira-Ramirez, Passivity-based Control of Euler-Lagrange Systems: Mechanical, Electrical and Electromechanical Applications, Springer Science \& Business Media, Berlin, Germany, 1998.

[8] R. Ortega, M. W. Spong, F. Gomez-Estern, and G. Blankenstein, "Stabilization of a class of underactuated mechanical systems via interconnection and damping assignment," IEEE Transactions on Automatic Control, vol. 47, no. 8, pp. 1218-1233, 2002.

[9] J. Hauser, S. Sastry, and P. Kokotovic, "Nonlinear control via approximate input-output linearization: the ball and beam example," IEEE Transactions on Automatic Control, vol. 37, no. 3, pp. 392-398, 1992.
[10] S. K. Agrawal and V. Sangwan, "Differentially flat designs of underactuated open-chain planar robots," IEEE Transactions on Robotics, vol. 24, no. 6, pp. 1445-1451, 2008.

[11] M. Fliess, J. Lévine, P. Martin, and P. Rouchon, "Flatness and defect of non-linear systems: introductory theory and examples," International Journal of Control, vol. 61, no. 6, pp. 1327-1361, 1995.

[12] H. Sira-Ramirez and S. Agrawal, Differentially Flat Systems, CRC Press, Boca Raton, FL, USA, 2004.

[13] J. Levine, Analysis and Control of Nonlinear Systems: A Flatness-Based Approach, Springer Science \& Business Media, Berlin, Germany, 2009.

[14] W. Amrhein, W. Gruber, W. Bauer, and M. Reisinger, "Magnetic levitation systems for cost-sensitive applicationssome design aspects," IEEE Transactions on Industry Applications, vol. 52, no. 5, pp. 3739-3752, 2016.

[15] I. Abed, N. Kacem, N. Bouhaddi, and M. Bouazizi, "Multimodal vibration energy harvesting approach based on nonlinear oscillator arrays under magnetic levitation," Smart Materials and Structures, vol. 25, no. 2, Article ID 025018, 2016.

[16] E. Shameli, M. B. Khamesee, and J. P. Huissoon, "Real-time control of a magnetic levitation device based on instantaneous modeling of magnetic field," Mechatronics, vol. 18, no. 10, pp. 536-544, 2008.

[17] E. Diller, J. Giltinan, G. Z. Lum, Z. Ye, and M. Sitti, "Sixdegree-of-freedom magnetic actuation for wireless microrobotics," The International Journal of Robotics Research, vol. 35, no. 1-3, pp. 114-128, 2016.

[18] S. Kim and K. Ishiyama, "Magnetic robot and manipulation for active-locomotion with targeted drug release," IEEE/ ASME Transactions on Mechatronics, vol. 19, no. 5, pp. 1651-1659, 2014.

[19] H. Lee, K. Kim, and J. Lee, "Review of maglev train technologies," IEEE Transactions on Magnetics, vol. 42, no. 7 , pp. 1917-1925, 2006.

[20] C. Drezet, N. Kacem, and N. Bouhaddi, "Design of a nonlinear energy harvester based on high static low dynamic stiffness for low frequency random vibrations," Sensors and Actuators A: Physical, vol. 283, pp. 54-64, 2018.

[21] S. Mahmoudi, N. Kacem, and N. Bouhaddi, "Enhancement of the performance of a hybrid nonlinear vibration energy harvester based on piezoelectric and electromagnetic transductions," Smart Materials and Structures, vol. 23, no. 7, Article ID 075024, 2014.

[22] Z. Zergoune, N. Kacem, and N. Bouhaddi, "On the energy localization in weakly coupled oscillators for electromagnetic vibration energy harvesting," Smart Materials and Structures, vol. 28, no. 7, Article ID 07LT02, 2019.

[23] B. Mann and N. Sims, "Energy harvesting from the nonlinear oscillations of magnetic levitation," Journal of Sound and Vibration, vol. 319, no. 1-2, pp. 515-530, 2009.

[24] Z.-J. Yang and M. Tateishi, "Adaptive robust nonlinear control of a magnetic levitation system," Automatica, vol. 37, no. 7, pp. 1125-1131, 2001.

[25] C. Knospe and E. Collins, "Special issue on magnetic bearing control," IEEE Transactions on Control Systems Technology, vol. 4, no. 5, p. 481, 1996.

[26] J.-H. Lee, P. E. Allaire, G. Tao, and X. Zhang, "Integral slidingmode control of a magnetically suspended balance beam: analysis, simulation, and experiment," IEEE/ASME Transactions on Mechatronics, vol. 6, no. 3, pp. 338-346, 2001.

[27] R. T. Rocha, J. M. Balthazar, A. M. Tusset, S. L. T. de Souza, F. C. Janzen, and H. C. Arbex, "On a non-ideal magnetic levitation system: nonlinear dynamical behavior and energy 
harvesting analyses," Nonlinear Dynamics, vol. 95, no. 4, pp. 3423-3438, 2019.

[28] J. D. Lindlau and C. R. Knospe, "Feedback linearization of an active magnetic bearing with voltage control," IEEE Transactions on Control Systems Technology, vol. 10, no. 1, pp. 21-31, 2002.

[29] A. D. Mahindrakar and V. Sankaranarayanan, "State-constrained stabilization of beam-balance systems," International Journal of Robust and Nonlinear Control, vol. 18, no. 3, pp. 333-350, 2008.

[30] T. Hu, Z. Lin, W. Jiang, and P. E. Allaire, "Constrained control design for magnetic bearing systems," Journal of Dynamic Systems, Measurement, and Control, vol. 127, no. 4, pp. 601616, 2005.

[31] H. Rodriguez, R. Ortega, and I. Mareels, "A novel passivitybased controller for an active magnetic bearing benchmark experiment," in Proceedings of the 2000 American Control Conference. ACC (IEEE Cat. No.00CH36334), vol. 3, pp. 2144-2148, IEEE, Chicago, IL, USA, June 2000.

[32] J. Lee, P. Allaire, G. Tao, J. Decker, and X. Zhang, "Experimental study of sliding mode control for a benchmark magnetic bearing system and artificial heart pump suspension," IEEE Transactions on Control Systems Technology, vol. 11, no. 1, pp. 128-138, 2003.

[33] J. Levine, J. Lottin, and J.-C. Ponsart, "A nonlinear approach to the control of magnetic bearings," IEEE Transactions on Control Systems Technology, vol. 4, no. 5, pp. 524-544, 1996.

[34] R. Morales and H. Sira-Ramírez, "Trajectory tracking for the magnetic ball levitation system via exact feedforward linearisation and gpi control," International Journal of Control, vol. 83, no. 6, pp. 1155-1166, 2010.

[35] R. Morales, V. Feliu, and H. Sira-Ramirez, "Nonlinear control for magnetic levitation systems based on fast online algebraic identification of the input gain," IEEE Transactions on Control Systems Technology, vol. 19, no. 4, pp. 757-771, 2011.

[36] A. R. Teel, "Global stabilization and restricted tracking for multiple integrators with bounded controls," Systems \& Control Letters, vol. 18, no. 3, pp. 165-171, 1992.

[37] S. Gayaka, L. Lu, and B. Yao, "Global stabilization of a chain of integrators with input saturation and disturbances: a new approach," Automatica, vol. 48, no. 7, pp. 1389-1396, 2012.

[38] S. K. Kannan and E. N. Johnson, "Adaptive control with a nested saturation reference model," in Proceedings of the AIAA Guidance, Navigation and Control Conference, pp. 1-11, Austin, TX, USA, August 2003.

[39] N. Marchand and A. Hably, "Global stabilization of multiple integrators with bounded controls," Automatica, vol. 41, no. 12, pp. 2147-2152, 2005.

[40] R. Lozano and D. D. Dimogianopoulos, "Stabilization of a chain of integrators with nonlinear perturbations: application to the inverted pendulum," in Proceedings. 42nd IEEE Conference on Decision and Control, IEEE, vol. 5, pp. 5191-5196, 2003.

[41] C. Barbu, R. Sepulchre, W. Lin, and P. V. Kokotovic, "Global asymptotic stabilization of the ball-and-beam system," in Proceedings of the 36th IEEE Conference on Decision and Control, vol. 3, pp. 2351-2355, IEEE, San Diego, CA, USA, December 1997.

[42] P. Castillo, R. Lozano, and A. Dzul, "Stabilization of a mini rotorcraft with four rotors," IEEE Control Systems, vol. 25, no. 6 , pp. $45-55,2005$.

[43] C. Aguilar-Ibañez, O. O. Gutiérrez-Frías, and M. S. SuárezCastañón, "Controlling the strongly damping inertia wheel pendulum via nested saturation functions," Computación y Sistemas, vol. 12, no. 4, 2009.

[44] A. Isidori, Nonlinear Control Systems, Springer, Berlin, Germany, 1995.

[45] C. A. Ibañez and O. G. Frias, "Controlling the inverted pendulum by means of a nested saturation function," Nonlinear Dynamics, vol. 53, no. 4, pp. 273-280, 2008.

[46] C. Aguilar Ibanez, J. C. Martinez Garcia, A. Soria Lopez, J. d. J. Rubio, and M. S. Suarez Castanon, "Stabilization of the inverted cart-pendulum system with linear friction," IEEE Latin America Transactions, vol. 16, no. 6, pp. 1650-1657, 2018.

[47] N. F. Al-Muthairi and M. Zribi, "Sliding mode control of a magnetic levitation system," Mathematical Problems in Engineering, vol. 2004, Article ID 657503, 15 pages, 2004.

[48] Z. Deng, J. Wang, J. Zheng et al., "Performance advances of hts maglev vehicle system in three essential aspects," IEEE Transactions on Applied Superconductivity, vol. 19, no. 3, pp. 2137-2141, 2009. 